\title{
Some geometric and analytic properties of solutions of Bernoulli free-boundary problems
}

\author{
EUGEN VARVARUCA ${ }^{\dagger}$ \\ Department of Mathematical Sciences, University of Bath, \\ Claverton Down, Bath BA2 7AY, United Kingdom
}

[Received 7 June 2006]

\begin{abstract}
A Bernoulli free-boundary problem is one of finding domains in the plane on which a harmonic function simultaneously satisfies linear homogeneous Dirichlet and inhomogeneous Neumann boundary conditions. For a general class of Bernoulli problems, we prove that any free boundary, possibly with many singularities, is necessarily the graph of a function. Also investigated are convexity and monotonicity properties of free boundaries. In addition, we obtain some optimal estimates on the gradient of the harmonic function in question.
\end{abstract}

\section{Introduction}

In this paper we investigate properties of solutions of Bernoulli free-boundary problems. The foundations for the study of this type of geometric problems, a specific example of which is the classical Stokes-wave problem in hydrodynamics, were laid out in a substantial paper of Shargorodsky and Toland [8].

A Bernoulli free-boundary problem $[8]$ is to find a locally rectifiable curve $\mathcal{S}:=\{(u(s), v(s))$ : $s \in \mathbb{R}\}$ in the $(X, Y)$-plane, where

$$
s \mapsto(u(s)-s, v(s)) \quad \text { is } 2 \pi \text {-periodic, }
$$

so that there exists a function $\psi$ harmonic in the domain $\Omega$ below $\mathcal{S}$ and continuous on $\bar{\Omega}$, such that

$$
\begin{aligned}
& \psi \quad \text { is } 2 \pi \text {-periodic in } X, \\
& \nabla \psi(X, Y) \rightarrow(0,1) \quad \text { as } Y \rightarrow-\infty, \text { uniformly in } X, \\
& \psi=0 \quad \text { on } \mathcal{S}, \\
& \frac{\partial \psi}{\partial n}=h(Y) \quad \text { almost everywhere on } \mathcal{S} .
\end{aligned}
$$

Here $n$ denotes the outward normal to $\Omega$ at points of $\mathcal{S}$, and $h$ is a given continuous nonnegative function. Since formally the tangential derivative of $\psi$ is zero almost everywhere on $\mathcal{S}$, the Neumann condition $1.1 \mathrm{e}^{\prime}$ has an equivalent reformulation as

$$
|\nabla \psi|^{2}=\lambda(Y) \quad \text { almost everywhere on } \mathcal{S}
$$

where $\lambda=h^{2}$. This condition is to be satisfied in a weak sense related to the theory of Hardy spaces (see [8, 12] for details). The object of investigation here will be the system of equations (1.1a)-(1.1e). Since the curve $\mathcal{S}$ is not prescribed a priori, it is called a free boundary.

\footnotetext{
${ }^{\dagger}$ E-mail: mapev@maths.bath.ac.uk
} 
The motivating example for the study of Bernoulli problems occurs in the theory of steady hydrodynamic waves, where $\lambda(r)=-2 g r$ for some positive constant $g$. In this context, the free boundary represents the profile of a Stokes wave, i.e. a steady periodic irrotational water wave of infinite depth, with a free surface under gravity and without surface tension (see [10]), $\psi$ is a stream function and $\left(\psi_{Y},-\psi_{X}\right)$ is a steady velocity field. Then $\left.1.1 \mathrm{~d}\right)$ and $(1.1 \mathrm{e})$ mean that $\mathcal{S}$ is a streamline at which the pressure in the flow is a constant. In hydrodynamics, a point on $\mathcal{S}$ where the velocity is zero is called a stagnation point.

For a solution $(\mathcal{S}, \psi)$ of $[1.1$, a point $(X, Y)$ on $\mathcal{S}$ is called a stagnation point if $\lambda(Y)=0$. The set $\mathcal{S}_{\mathcal{N}}$ of stagnation points is closed and has zero measure on $\mathcal{S}$ (see [8]), and it is only at stagnation points that the free boundary need not be smooth. Indeed, $\mathcal{S} \backslash \mathcal{S}_{\mathcal{N}}$ is a union of smooth arcs, whose precise degree of regularity depends on that of $\lambda$ (see [5, 12]). The nature of the singularities of free boundaries at stagnation points was investigated in [12]. The conclusion was that in many situations (but not always) a free boundary which is symmetric with respect to a vertical line passing through an isolated singular point must have a corner at that point, whose size depends on the nonlinearity $\lambda$. That was an extension of the result proved in [2, 6] that symmetric Stokes waves of extreme form have a corner of $120^{\circ}$.

In this paper we investigate some geometric properties of free boundaries, whether singular or not. Note that a priori $\mathcal{S}$ might have a very complicated shape, especially if there are stagnation points; in particular, it is not assumed a priori that $\mathcal{S}$ is the graph of a function. We also establish some optimal estimates on the gradient of the harmonic function $\psi$. There follows a brief description of the main results obtained.

A preliminary observation is that there exists $Y_{0}$ with $h\left(Y_{0}\right)=1$ such that $\mathcal{S}$ intersects the horizontal line $Y=Y_{0}$; when $h$ is a strictly decreasing function, such a $Y_{0}$ is unique. Some of our results refer to the shape of the part of $\mathcal{S}$ which lies either above or below the line $Y=Y_{0}$. Note that $\mathcal{S}_{0}:=\left\{\left(X, Y_{0}\right): X \in \mathbb{R}\right\}$ and $\psi_{0}(X, Y):=Y-Y_{0}$ provide a solution of (1.1), which we call a trivial solution.

Then, under the assumption that $\log h$ is decreasing and concave, we show that

- $\mathcal{S}$ must be globally the graph of a function, irrespective of the number of stagnation points on $\mathcal{S}$.

This was previously known [8] only when there were at most countably many stagnation points on $\mathcal{S}$, although it should be said that the result in [8] is different and more general in some other respects, as we explain in detail later. The proof here is partly based on a differential inequality from [8], but avoids some rather intricate estimates needed there. Instead, the remaining part is based on geometrical considerations inspired by the argument of Spielvogel [9], who was the first to prove this result for the case of smooth solutions of the water-wave problem. (However, it is our impression that the argument in [9] is vague and lacks sufficient rigor, and in fact the claim in the penultimate paragraph of [9. Proof of Theorem 3b] is incorrect. Furthermore, in the case considered here there are significant additional difficulties due to the presence of the stagnation points.)

We also generalize this result by showing that, if the above assumption on $h$ is relaxed to a local one, then, roughly speaking,

- a curve $\mathcal{S}$ with stagnation points may fail to be a graph only at a positive distance from the set $\mathcal{S}_{\mathcal{N}}$.

Suppose again that $\log h$ is globally decreasing and concave, and let $\mathcal{S}:=\{(X, \eta(X)): X \in \mathbb{R}\}$, where $\eta: \mathbb{R} \rightarrow \mathbb{R}$ is continuous and $2 \pi$-periodic. We prove that

- $\eta$ is a convex function of $X$ on any interval on which $\eta<Y_{0}$, where $h\left(Y_{0}\right)=1$, 
- if $X_{1}, X_{2}$ are such that $\eta^{\prime}\left(X_{1}\right)=\eta^{\prime}\left(X_{2}\right)=0$ and $\eta^{\prime}(X) \neq 0$ for all $X \in\left(X_{1}, X_{2}\right)$, then

$$
\left|\nabla \psi\left(X_{1}, \eta\left(X_{1}\right)\right)\right|+\left|\nabla \psi\left(X_{2}, \eta\left(X_{2}\right)\right)\right| \leqslant 2 .
$$

The convexity result is new even for the water-wave problem. Surprisingly perhaps, the estimate (1.2) was noted in passing by Toland [10, (x), p. 15] for a restricted class of solutions of the waterwave problem, but here we emphasize its generality and its independence from $h$, and we give a different and more transparent proof. Note that $[1.2]$ implies that $|\nabla \psi| \leqslant 2$ everywhere on $\mathcal{S}$, and hence by the maximum principle

$$
|\nabla \psi| \leqslant 2 \quad \text { everywhere in } \Omega .
$$

We also give some new results in the case when $\log h$ is decreasing and convex. For a curve $\mathcal{S}$ which is the graph of a function $\eta$,

- if $X_{1}, X_{2}$ are such that $\eta^{\prime}\left(X_{1}\right)=\eta^{\prime}\left(X_{2}\right)=0$ and $\eta^{\prime}(X) \neq 0$ for all $X \in\left(X_{1}, X_{2}\right)$, then

$$
\left|\nabla \psi\left(X_{1}, \eta\left(X_{1}\right)\right)\right|+\left|\nabla \psi\left(X_{2}, \eta\left(X_{2}\right)\right)\right| \geqslant 2,
$$

- $\eta$ does not have any strict local minima on any interval on which $\eta>Y_{0}$, where $h\left(Y_{0}\right)=1$.

A family of explicit solutions of $(1.1)$ is used to show that the estimates $(1.2)-(1.4)$ are sharp.

All the preceding results are derived from corresponding results about solutions of the nonlinear pseudo-differential equation [2.5, an equation first derived in [8], the study of which is equivalent to that of (1.1) up to the fact that a certain curve in the plane is non-self-intersecting. A new sufficient condition for this non-self-intersection property is also given.

\section{Bernoulli free boundaries}

We start by collecting notation and recalling some notions and classical results from harmonic analysis in the unit disc (see [3, 4] for more details).

We denote by $L_{2 \pi}^{p}, 0<p<\infty$, the space of $2 \pi$-periodic locally $p^{\text {th }}$-power summable realvalued functions, and by $L_{2 \pi}^{\infty}$ the space of $2 \pi$-periodic essentially bounded real-valued functions. For $1 \leqslant p \leqslant \infty$, let $W_{2 \pi}^{1, p}$ be the space of absolutely continuous $2 \pi$-periodic functions $u$ with weak first derivatives $u^{\prime} \in L_{2 \pi}^{p}$. For any $u \in L_{2 \pi}^{1}$, the conjugate function $\mathcal{C} u$ is defined almost everywhere as a Cauchy principal value integral

$$
\mathcal{C} u(x)=\frac{1}{2 \pi} \int_{-\pi}^{\pi} \cot \left(\frac{1}{2}(x-y)\right) u(y) \mathrm{d} y .
$$

For any $p \in(1, \infty), \mathcal{C}$ maps $L_{2 \pi}^{p}$ into itself. $\mathcal{C}$ does not map $L_{2 \pi}^{1}$ into itself but, if $u \in L_{2 \pi}^{1}$, then $\mathcal{C} u \in L_{2 \pi}^{p}$ for all $p \in(0,1)$. Let $\mathcal{H}_{\mathbb{R}}^{1}$ be the real Hardy space of functions $u \in L_{2 \pi}^{1}$ with $\mathcal{C} u \in L_{2 \pi}^{1}$, and let also $\mathcal{H}_{\mathbb{R}}^{1,1}:=\left\{u \in W_{2 \pi}^{1,1}: u^{\prime} \in \mathcal{H}_{\mathbb{R}}^{1}\right\}$.

Let $D$ denote the open unit disc centred at 0 in the complex plane. For a holomorphic function $U: D \rightarrow \mathbb{C}$, let $U_{r}(t)=U\left(r e^{i t}\right)$ for $t \in \mathbb{R}$ and $r \in(0,1)$. For $0<p \leqslant \infty$, the Hardy class $\mathcal{H}_{\mathbb{C}}^{p}$ is the set of holomorphic functions $U: D \rightarrow \mathbb{C}$ such that $\sup _{r \in(0,1)}\left\|U_{r}\right\|_{L_{2 \pi}^{p}}<\infty$. If $U \in \mathcal{H}_{\mathbb{C}}^{p}$, $0<p \leqslant \infty$, then $U^{*}(t):=\lim _{r \nearrow 1} U_{r}(t)$ is well defined almost everywhere, satisfies $U^{*} \in L_{2 \pi}^{p}$, and $\log \left|U^{*}\right| \in L_{2 \pi}^{1}$ if $U \not \equiv 0$. 
We now recall from [8] how the study of the free-boundary problem $[1.1]$ is equivalent to the study of certain nonlinear pseudo-differential equations for periodic functions of one real variable.

Throughout the paper, the continuous functions $h, \lambda: I \rightarrow[0, \infty)$ are related by $h=\sqrt{\lambda}$ on $I$, where either

$$
I=\mathbb{R} \quad \text { and } \quad h>0 \text { on } \mathbb{R},
$$

or

$$
I=(-\infty, \omega], \omega \in \mathbb{R}, \quad \text { and } \quad h(\omega)=0, h>0 \text { on }(-\infty, \omega) .
$$

Let

$$
I_{0}:=\{r \in I: h(r)>0\} .
$$

We also assume that

$$
h \in C_{\text {loc }}^{1, \alpha}\left(I_{0}\right) \quad \text { for some } \alpha \in(0,1) .
$$

When $(2.1)$ holds, there can be no stagnation points on $\mathcal{S}$.

When (2.2) holds, stagnation points, if they exist, must be located on the line $Y=\omega$, and are necessarily points of maximum height on $\mathcal{S}$. There is no loss of generality in assuming throughout that $\omega=0$. Indeed, if $\omega \neq 0$, then one can replace $h$ by $h_{\omega}$, where $h_{\omega}(r)=h(r+\omega), \mathcal{S}$ by $\mathcal{S}_{\omega}:=\left\{\left(u_{\omega}(s), v_{\omega}(s)\right): s \in \mathbb{R}\right\}$, where $u_{\omega}(s)=u(s), v_{\omega}(s)=v(s)-\omega$, and $\psi$ by $\psi_{\omega}$ given by $\psi_{\omega}(X, Y)=\psi(X, Y+\omega)$ to get a new solution of 1.1 .

Any solution $(\mathcal{S}, \psi)$ of 1.1 gives rise to a function $w \in \mathcal{H}_{\mathbb{R}}^{1,1}$ satisfying

$$
\lambda(w)\left\{w^{\prime 2}+\left(1+\mathcal{C} w^{\prime}\right)^{2}\right\}=1 \quad \text { almost everywhere, }
$$

and with the property that, if $W \in \mathcal{H}_{\mathbb{C}}^{1}$ is such that

$$
W^{*}=w^{\prime}+i\left(1+\mathcal{C} w^{\prime}\right),
$$

then

$$
1 / W \in \mathcal{H}_{\mathbb{C}}^{\infty}
$$

The curve $\mathcal{S}$ can then be expressed in parametric form as

$$
\mathcal{S}=\{(-(\gamma+t+\mathcal{C} w(t)), w(t)): t \in \mathbb{R}\},
$$

where $\gamma \in \mathbb{R}$ is a constant. Hence

$$
t \mapsto(-(t+\mathcal{C} w(t)), w(t)) \quad \text { is injective on } \mathbb{R} .
$$

Let

$$
\mathcal{N}:=\{t \in \mathbb{R}: h(w(t))=0\} .
$$

Then the set of stagnation points on $\mathcal{S}$ is given by

$$
\mathcal{S}_{\mathcal{N}}=\{(-(\gamma+t+\mathcal{C} w(t)), w(t)): t \in \mathcal{N}\} .
$$

In what follows, the elements of $\mathcal{N}$ will also be referred to as stagnation points. The set $\mathcal{N}$ is closed and has zero measure. 
Conversely, suppose that $w \in \mathcal{H}_{\mathbb{R}}^{1,1}$ satisfies 2.5 and that 2.7 holds. Let $\mathcal{S}$ be defined by 2.6, where $\gamma \in \mathbb{R}$, and let $\Omega$ be the domain below $\mathcal{S}$. Then there exists a conformal mapping $\varpi$ of $\Omega$ onto $\mathbb{C}_{-}$such that, if $\psi=\operatorname{Im} \varpi$, then $(\mathcal{S}, \psi)$ is a solution of $(1.1)$.

Following [8], we now derive further properties of solutions of 2.5. If $w \in \mathcal{H}_{\mathbb{R}}^{1,1}$ satisfies 2.5, then $1 / h(w) \in L_{2 \pi}^{1}$ and $h(w) \in L_{2 \pi}^{\infty}$. One can define $2 \pi$-periodic functions $\tau, \vartheta$ by

$$
\begin{aligned}
\tau & :=-\log h(w), \\
\vartheta & :=-\mathcal{C} \tau,
\end{aligned}
$$

where $\tau, \vartheta \in L_{2 \pi}^{p}$ for all $p \in(1, \infty)$, and $\tau, \vartheta$ have zero mean. It also follows that

$$
\begin{aligned}
h(w) w^{\prime} & =\sin \vartheta, \\
h(w)\left(1+\mathcal{C} w^{\prime}\right) & =\cos \vartheta .
\end{aligned}
$$

Observe now from $(2.10)$ that, if $\mathcal{S}$ represents a free boundary parametrized by $(2.6)$, then, for almost all $t \in \mathbb{R},-\vartheta(t)$ represents the angle which the tangent to $\mathcal{S}$ at the point $(-(\gamma+t+\mathcal{C} w(t)), w(t))$ makes with the horizontal.

PROPOSITION 2.1 Let $(\mathcal{S}, \psi)$ be a non-trivial solution of 1.1 . Let $Y_{c}:=\max \{v(s): s \in \mathbb{R}\}$ and $Y_{t}:=\min \{v(s): s \in \mathbb{R}\}$. Then $h\left(Y_{c}\right)<1$ and $h\left(Y_{t}\right)>1$.

Corollary 2.2 In the notation of Proposition 2.1. there exists $Y_{0} \in\left(Y_{t}, Y_{c}\right)$ such that $h\left(Y_{0}\right)=1$.

Proof of Proposition 2.1] Note first that, by [8, Proof of Theorem 2.7], there exists $d \in \mathbb{R}$ such that

$$
\psi(X, Y)-Y-d \rightarrow 0 \quad \text { as } Y \rightarrow-\infty, \text { uniformly in } X .
$$

Also, since 2.4 holds, [12, Theorem 2.3] shows that $\mathcal{S} \backslash \mathcal{S}_{\mathcal{N}}$ is a union of $C_{\text {loc }}^{2, \alpha}$ curves, $\psi \in$ $C_{\text {loc }}^{2, \alpha}\left(\Omega \cup\left(\mathcal{S} \backslash \mathcal{S}_{\mathcal{N}}\right)\right)$ and

$$
\frac{\partial \psi}{\partial n}=h(Y) \quad \text { everywhere on } \mathcal{S} \backslash \mathcal{S}_{\mathcal{N}}
$$

Consider now the function $\xi$ in $\Omega$ given by

$$
\xi(X, Y):=\psi(X, Y)-Y \quad \text { for all }(X, Y) \in \Omega .
$$

Then $\xi$ is a bounded harmonic function in $\Omega$. Since $\psi=0$ on $\mathcal{S}$, it follows that $-Y_{c} \leqslant \xi \leqslant-Y_{t}$ on $\mathcal{S}$ and hence, by the maximum principle, $-Y_{c}<\xi<-Y_{t}$ in $\Omega$.

Let $X_{c}$ and $X_{t}$ be such that $\left(X_{c}, Y_{c}\right) \in \mathcal{S}$ and $\left(X_{t}, Y_{t}\right) \in \mathcal{S}$. Then $\xi$ attains its extrema in $\bar{\Omega}$ at these points. By the Hopf boundary-point lemma,

$$
0<\xi_{Y}\left(X_{t}, Y_{t}\right)=\psi_{Y}\left(X_{t}, Y_{t}\right)-1,
$$

and hence $h\left(Y_{t}\right)>1$ by 2.12]. If $\mathcal{S}_{\mathcal{N}}=\emptyset$, then similarly

$$
0>\xi_{Y}\left(X_{c}, Y_{c}\right)=\psi_{Y}\left(X_{c}, Y_{c}\right)-1,
$$

and $h\left(Y_{c}\right)<1$. If $\mathcal{S}_{\mathcal{N}} \neq \emptyset$, then 2.2 holds, and hence $h\left(Y_{c}\right)=0<1$. This completes the proof of Proposition 2.1 


\section{The main results}

Since solutions $w$ of 2.5 give rise to solutions $(\mathcal{S}, \psi)$ of (1.1) only if (2.7) holds, it is of interest to exhibit situations when (2.7) follows automatically from 2.5). Under the assumption that

$h$ is strictly decreasing and $\log h$ is concave on $I_{0}$,

it was proved in [8, Theorem 3.5] that solutions $w$ of $[2.5$ for which $\mathcal{N}$ is at most denumerable satisfy

$$
\vartheta(t) \in(-\pi / 2, \pi / 2) \quad \text { for almost all } t \in \mathbb{R},
$$

and hence

$$
1+\mathcal{C} w^{\prime}(t)>0 \quad \text { for almost all } t \in \mathbb{R} \text {. }
$$

By (2.10), 3.3) ensures that 2.7] holds and, moreover, $\mathcal{S}$ is globally a graph, in the sense that there exists a continuous $2 \pi$-periodic function $\eta: \mathbb{R} \rightarrow \mathbb{R}$ such that

$$
\mathcal{S}=\{(X, \eta(X)): X \in \mathbb{R}\} .
$$

Here we prove that, under the assumption 3.1 , free boundaries must necessarily be global graphs, irrespective of the number of stagnation points.

THEOREM 3.1 Suppose that $h$ satisfies (3.1), and let $(\mathcal{S}, \psi)$ be a solution of (1.1). Then $\mathcal{S}$ is of the form (3.4) for some continuous $2 \pi$-periodic function $\eta: \mathbb{R} \rightarrow \mathbb{R}$.

Theorem 3.1 is an immediate consequence of the following result.

THEOREM 3.2 Suppose that $h$ satisfies (3.1). Let $w$ be a solution of 2.5) such that 2.7p holds. Then $w$ satisfies (3.3).

The key point here is that the assumptions of Theorems 3.1 and 3.2 admit the possibility that the set of stagnation points might be nondenumerable. This was not the case in [8, Theorem 3.5], where the denumerability of $\mathcal{N}$ was crucial. On the other hand, in [8] the fact that (2.7p holds was not an assumption, but a conclusion. Therefore Theorem 3.2 is neither weaker nor stronger than, but rather complementary to, [8, Theorem 3.5].

The next result is a local version of Theorem 3.2 for solutions $w$ of 2.5 for which $\mathcal{N} \neq \emptyset$, where (2.2) holds with $\omega=0$. The aim is to determine intervals in $\mathbb{R} \backslash \mathcal{N}$ on which

$$
1+\mathcal{C} w^{\prime}>0 .
$$

THEOREM 3.3 Suppose that there exists $\delta \in(-\infty, 0)$ such that

$$
h \text { is strictly decreasing and } \log h \text { is concave on }[\delta, 0) \text {. }
$$

Let $w$ be a solution of (2.5) which satisfies (2.7) and for which $\mathcal{N} \neq \emptyset$. Then there exists $\beta \in[\delta, 0$ ), which depends only on $\min \mathcal{R}(w)$, such that, for any $a, b \in \mathcal{N}$ with $(a, b) \subset \mathbb{R} \backslash \mathcal{N}$, the following hold.

(i) If $w(t) \in[\beta, 0)$ for all $t \in(a, b)$, then 3.5 holds everywhere on $(a, b)$.

(ii) If $d \in(a, b)$ is such that $w(d)=\beta$ and $w(t) \in[\beta, 0)$ for all $t \in[d, b)$, then (3.5) holds everywhere on $[d, b)$. 
(iii) If $c \in(a, b)$ is such that $w(c)=\beta$ and $w(t) \in[\beta, 0)$ for all $t \in(a, c]$, then 3.5 ) holds everywhere on $(a, c]$.

Theorem 3.3 means that for any solution of (1.1) there exists a horizontal strip, determined by the lines $Y=\beta$ and $Y=0$, such that any arc of $\mathcal{S} \backslash \mathcal{S}_{\mathcal{N}}$ contained in this strip and having end-points on either of these two lines, but at least one end-point on the line $Y=0$, is necessarily the graph of a function. In particular, if $\mathcal{S}$ has infinitely many stagnation points on a period, then the local rectifiability of $\mathcal{S}$ shows that, among the countably many arcs of $\mathcal{S} \backslash \mathcal{S}_{\mathcal{N}}$ with endpoints in $\mathcal{S}_{\mathcal{N}}$, all but finitely many on a period of $\mathcal{S}$ are contained in such a strip, and therefore are necessarily graphs.

The next result can be used to deduce another geometric property of free boundaries.

Proposition 3.4 Suppose that $h$ satisfies (3.1) and $w$ satisfies (2.5). Let $s_{1}, s_{2}$ with $s_{1}<s_{2}$ be such that $h\left(w\left(s_{1}\right)\right)=h\left(w\left(s_{2}\right)\right)=1$ and $h(w(s))>1$ for all $s \in\left(s_{1}, s_{2}\right)$. Then $\vartheta^{\prime}>0$ on $\left(s_{1}, s_{2}\right)$.

Indeed, for $\mathcal{S}$ of the form (3.4), let $Y_{0} \in\left(Y_{t}, Y_{c}\right)$ be such that $h\left(Y_{0}\right)=1$, given by Corollary 2.2. Proposition 3.4 shows that, if $X_{1}, X_{2}$ with $X_{1}<X_{2}$ are such that $\eta\left(X_{1}\right)=\eta\left(X_{2}\right)=Y_{0}$ and $\eta(X)<Y_{0}$ for all $X \in\left(X_{1}, X_{2}\right)$, then $\eta$ is a convex function on the interval $\left(X_{1}, X_{2}\right)$. A much more difficult problem would be to exhibit situations in which any smooth arc of $\mathcal{S}$ joining two stagnation points is the graph of a convex function; the only result currently known is [7], that there exist such solutions when $h(r)=c(-r)^{\alpha}$, where $c>0$ and $\alpha \in[1 / 2, \infty)$.

The following result provides bounds for solutions of (2.5) when 3.1 holds.

THEOREM 3.5 Suppose that $h$ satisfies (3.1), and let $w$ be a solution of (2.5) for which (3.2) holds. Let $t_{1}, t_{2}$ be such that $\left(t_{1}, t_{2}\right) \subset \mathbb{R} \backslash \mathcal{N}$.

(i) Suppose that $t_{1}, t_{2} \in \mathbb{R} \backslash \mathcal{N}, w^{\prime}\left(t_{1}\right)=w^{\prime}\left(t_{2}\right)=0$, and either $0 \not \equiv w^{\prime} \geqslant 0$ on $\left(t_{1}, t_{2}\right)$, or $0 \not \equiv w^{\prime} \leqslant 0$ on $\left(t_{1}, t_{2}\right)$. Then

$$
h\left(w\left(t_{1}\right)\right)+h\left(w\left(t_{2}\right)\right) \leqslant 2 .
$$

(ii) Suppose that $t_{1} \in \mathbb{R} \backslash \mathcal{N}, t_{2} \in \mathcal{N}, w^{\prime}\left(t_{1}\right)=0,0 \not \equiv w^{\prime} \geqslant 0$ on $\left(t_{1}, t_{2}\right)$. Then $h\left(w\left(t_{1}\right)\right) \leqslant 2$.

(iii) Suppose that $t_{1} \in \mathcal{N}, t_{2} \in \mathbb{R} \backslash \mathcal{N}, w^{\prime}\left(t_{1}\right)=0,0 \neq w^{\prime} \leqslant 0$ on $\left(t_{1}, t_{2}\right)$. Then $h\left(w\left(t_{2}\right)\right) \leqslant 2$.

Note that in Theorem 3.5 the requirement that $(3.2)$ holds is an extremely weak restriction when $h$ is decreasing and $\log h$ is concave, since by [8, Theorem 3.5] and Theorem 3.2 it is satisfied automatically for all solutions $w$ of 2.5 with at most countably many stagnation points, as well as for those, with any number of stagnation points, which correspond to free boundaries.

Corollary 3.6 Suppose that $h$ satisfies (3.1), and let $w$ be a solution of (2.5) for which (3.2) holds. Then $h(w(t)) \leqslant 2$ for all $t \in \mathbb{R}$.

When interpreted in terms of free boundaries, Theorem 3.5 and Corollary 3.6 lead to the estimates (1.2) and (1.3).

We now give some new results in the case when (2.1) holds and

$h$ is strictly decreasing and $\log h$ is convex on $\mathbb{R}$.

The following result is an analogue of Theorem 3.5 when $(3.8)$ holds instead of 3.1 .

THEOREM 3.7 Suppose that $h$ satisfies (3.8), and let $w$ be a solution of (2.5) for which (3.2) holds. Let $t_{1}, t_{2}$ with $t_{1}<t_{2}$ be such that $w^{\prime}\left(t_{1}\right)=w^{\prime}\left(t_{2}\right)=0$, and either $0 \not \equiv w^{\prime} \geqslant 0$ on $\left(t_{1}, t_{2}\right)$, or $0 \not \equiv w^{\prime} \leqslant 0$ on $\left(t_{1}, t_{2}\right)$. Then

$$
h\left(w\left(t_{1}\right)\right)+h\left(w\left(t_{2}\right)\right) \geqslant 2 .
$$


Corollary 3.8 Suppose that $h$ satisfies (3.8), and let $w$ be a solution of 2.5 for which 3.2 holds. Let $s_{1}, s_{2}$ with $s_{1}<s_{2}$ be such that $h\left(w\left(s_{1}\right)\right)=h\left(w\left(s_{2}\right)\right)=1$ and $h(w(s))<1$ for all $s \in\left(s_{1}, s_{2}\right)$. Then $w$ does not have any strict local minima in $\left(s_{1}, s_{2}\right)$.

In terms of free boundaries, Theorem 3.7 leads to the estimate 1.4 , while Corollary 3.8 has an obvious interpretation.

The next result gives a sufficient condition for solutions of (2.5) to give rise to free boundaries when $h$ satisfies 3.8.

TheOREM 3.9 Suppose that $h$ satisfies (3.8), and let $w$ be a solution of 2.5 ) such that $h(w(t)) \leqslant 2$ for all $t \in \mathbb{R}$. Then 3.2 holds, and hence so does 3.3.

We conclude this section by showing, by means of a family of examples of explicit solutions of 2.5, that the results of Theorem 3.5, Corollary 3.6 and Theorem 3.7 are sharp, at least for nonsingular solutions. The examples below are obtained by slightly modifying those in [8, Subsection 2.9.1]. Let $\lambda: \mathbb{R} \rightarrow(0, \infty)$ be given by $\lambda(r)=e^{-2 r}$ for all $r \in \mathbb{R}$, and let $h:=\sqrt{\lambda}$. For $0 \leqslant b<1$, let

$$
w_{b}(t)=-\frac{1}{2} \log \left(1+b^{2}+2 b \cos t\right) \quad \text { for all } t \in \mathbb{R} .
$$

Then $w_{b}$ is a non-singular solution of 2.5. Since $\log h$ is decreasing and affine on $\mathbb{R}, h$ satisfies the assumptions of both Theorems 3.5 and 3.7 . Note that, for all $t \in \mathbb{R}$,

$$
\lambda\left(w_{b}(t)\right)=1+b^{2}+2 b \cos t
$$

and $w_{b}^{\prime}(t)=0$ if and only if $t=n \pi$ for some $n \in \mathbb{Z}$. It is immediate from 3.11 that, for all $n \in \mathbb{Z}$,

$$
h\left(w_{b}(n \pi)\right)+h\left(w_{b}((n+1) \pi)\right)=2,
$$

hence the results of Theorems 3.5 and 3.7 are sharp. Also, since

$$
\max \left\{h\left(w_{b}(t)\right): t \in \mathbb{R}\right\}=1+b \quad \text { for all } b \in[0,1),
$$

it follows that the result of Corollary 3.6 is also sharp.

\section{Proofs of the main results}

We now give the proofs of the results in the previous section. We start with general considerations which are relevant for all the proofs.

If $J$ is any open interval contained in $\mathbb{R} \backslash \mathcal{N}$ then, since $(2.4)$ holds, it follows from [12, Theorem 2.1] that $w \in C_{\mathrm{loc}}^{2, \alpha}(J)$. It is proved in [8] that

$$
\begin{aligned}
& (\mathcal{C} \log h(w))^{\prime}(t)-\frac{h^{\prime}(w(t))}{h(w(t))} \mathcal{C} w^{\prime}(t) \\
& \quad=\frac{1}{4 \pi} \int_{-\pi}^{\pi} \frac{\log h(w(t))+\left(h^{\prime}(w(t)) / h(w(t))\right)(w(s)-w(t))-\log h(w(s))}{\sin ^{2} \frac{t-s}{2}} \mathrm{~d} s .
\end{aligned}
$$

The formula (4.1), which is just an identity based on the definition of a conjugate function, is valid for all $t \in J$ under the present regularity assumptions on $h$ and $w$. Since $J \subset \mathbb{R} \backslash \mathcal{N}$ was arbitrary, (4.1) is valid everywhere on $\mathbb{R} \backslash \mathcal{N}$. 
One can now see that, if $w$ is a solution of $\left[2.5\right.$, then $\vartheta \in C_{\text {loc }}^{1, \alpha}(\mathbb{R} \backslash \mathcal{N})$ and, for all $t \in \mathbb{R} \backslash \mathcal{N}$,

$$
\vartheta^{\prime}(t)-\frac{h^{\prime}(w(t))}{h(w(t))}\left(\frac{\cos \vartheta(t)}{h(w(t))}-1\right) \geqslant 0 \quad \text { if } \log h \text { is concave, }
$$

and

$$
\vartheta^{\prime}(t)-\frac{h^{\prime}(w(t))}{h(w(t))}\left(\frac{\cos \vartheta(t)}{h(w(t))}-1\right) \leqslant 0 \quad \text { if } \log h \text { is convex. }
$$

The estimate (4.2) is one of the key ingredients in the proof of [8, Theorem 3.5]. See also [11] for smooth solutions of the water-wave problem.

Proof of Proposition 3.4 It follows from (3.1) that $h^{\prime}<0$ on $I_{0}$. Using also the fact that $\cos \vartheta(s)<$ $h(w(s))$ for all $s \in\left(s_{1}, s_{2}\right)$, it is immediate from (4.2) that $\vartheta^{\prime}>0$ on $\left(s_{1}, s_{2}\right)$.

At the heart of the proofs of Theorems 3.5, 3.7 and 3.9 lies the combination of $4.2 / \sqrt{4.3}$ with the following new algebraic identity satisfied by solutions of 2.5 everywhere on $\mathbb{R} \backslash \mathcal{N}$ :

$$
\begin{aligned}
\frac{\mathrm{d}}{\mathrm{d} t}\left(\frac{h^{2}(w)}{2}-h(w) \cos \vartheta\right) & =h(w) h^{\prime}(w) w^{\prime}-h^{\prime}(w) w^{\prime} \cos \vartheta+h(w) \sin \vartheta \vartheta^{\prime} \\
& =w^{\prime} h^{2}(w)\left\{\vartheta^{\prime}-\frac{h^{\prime}(w)}{h(w)}\left(\frac{\cos \vartheta}{h(w)}-1\right)\right\} .
\end{aligned}
$$

Proof of Theorem 3.5. Suppose first that we are in the situation (i), and with $0 \neq \equiv w^{\prime} \geqslant 0$ on $\left(t_{1}, t_{2}\right)$. It follows from (4.4) using (4.2) that

$$
t \mapsto \frac{h^{2}(w(t))}{2}-h(w(t)) \cos \vartheta(t) \quad \text { is increasing on }\left(t_{1}, t_{2}\right) .
$$

Since $\vartheta \in(-\pi / 2, \pi / 2)$, it follows that $\vartheta\left(t_{1}\right)=\vartheta\left(t_{2}\right)=0$. Hence we deduce from $(4.5)$ that

$$
\frac{h^{2}\left(w\left(t_{1}\right)\right)}{2}-h\left(w\left(t_{1}\right)\right) \leqslant \frac{h^{2}\left(w\left(t_{2}\right)\right)}{2}-h\left(w\left(t_{2}\right)\right),
$$

or, equivalently,

$$
\left(h\left(w\left(t_{1}\right)\right)-h\left(w\left(t_{2}\right)\right)\right)\left(h\left(w\left(t_{1}\right)\right)+h\left(w\left(t_{2}\right)\right)-2\right) \leqslant 0 .
$$

But $w\left(t_{1}\right)<w\left(t_{2}\right)$ by assumption, and hence, since $h$ is strictly decreasing, $h\left(w\left(t_{1}\right)\right)>h\left(w\left(t_{2}\right)\right)$. The required conclusion follows from (4.6).

Suppose now that we are in the situation (i), but with $0 \not \equiv w^{\prime} \leqslant 0$ on $\left(t_{1}, t_{2}\right)$. Instead of 4.5 , we now have that

$$
t \mapsto \frac{h^{2}(w(t))}{2}-h(w(t)) \cos \vartheta(t) \quad \text { is decreasing on }\left(t_{1}, t_{2}\right),
$$

and the required conclusion follows by an argument similar to that of the previous case.

Suppose now that we are in the situation (ii). It follows as above that, for all $t \in\left(t_{1}, t_{2}\right)$,

$$
\frac{h^{2}\left(w\left(t_{1}\right)\right)}{2}-h\left(w\left(t_{1}\right)\right) \leqslant \frac{h^{2}(w(t))}{2}-h(w(t)) \cos \vartheta(t) .
$$

The required conclusion is obtained by passing to the limit in $(4.8)$ as $t \nearrow t_{2}$, using the fact that $h(w(t)) \rightarrow 0$ as $t \nearrow t_{2}$, since $t_{2} \in \mathcal{N}$. 3.5 .

The analysis in the situation (iii) is entirely analogous. This completes the proof of Theorem 
Proof of Corollary 3.6 Recall that $\tau$ has zero mean, where $\tau=-\log h(w)$. If $h(w) \equiv c$, where $c>0$ is a constant, then necessarily $c=1$. Suppose that $h(w) \not \equiv c$. Let $t_{1} \in \mathbb{R}$ be such that

$$
w\left(t_{1}\right)=\min \{w(t): t \in \mathbb{R}\} .
$$

Then $w^{\prime}\left(t_{1}\right)=0$ and $h\left(w\left(t_{1}\right)\right)>1$. Since $h$ is decreasing, it suffices to prove that $h\left(w\left(t_{1}\right)\right) \leqslant 2$. Let $\tilde{t}>t_{1}$ be such that $h(w(\tilde{t}))=1$ and $h(w(t))>1$ for all $t \in\left(t_{1}, \tilde{t}\right)$. By Proposition 3.4 . $\vartheta^{\prime}(t) \geqslant 0$ for all $t \in\left(t_{1}, \tilde{t}\right)$. Since $\vartheta\left(t_{1}\right)=0$, it follows that $\vartheta(t) \geqslant 0$ for all $t \in\left(t_{1}, \tilde{t}\right)$, and therefore $0 \not \equiv w^{\prime} \geqslant 0$ on $\left(t_{1}, \tilde{t}\right)$. There exists $t_{2}>t_{1}$ with $\left(t_{1}, t_{2}\right) \subset \mathbb{R} \backslash \mathcal{N}$ such that $w^{\prime} \geqslant 0$ on $\left(t_{1}, t_{2}\right)$, and either $t_{2} \in \mathbb{R} \backslash \mathcal{N}$ and $w^{\prime}\left(t_{2}\right)=0$, or $t_{2} \in \mathcal{N}$. In either case we conclude from Theorem 3.5 that $h\left(w\left(t_{1}\right)\right) \leqslant 2$, which is the required result.

Proof of Theorem 3.7. It suffices to consider the case when $0 \neq \equiv w^{\prime} \geqslant 0$ on $\left(t_{1}, t_{2}\right)$, since the other case can be treated in an entirely similar way. It follows from (4.4) using (4.3) that

$$
t \mapsto \frac{h^{2}(w(t))}{2}-h(w(t)) \cos \vartheta(t) \quad \text { is decreasing on }\left(t_{1}, t_{2}\right),
$$

so that

$$
\frac{h^{2}\left(w\left(t_{1}\right)\right)}{2}-h\left(w\left(t_{1}\right)\right) \geqslant \frac{h^{2}\left(w\left(t_{2}\right)\right)}{2}-h\left(w\left(t_{2}\right)\right),
$$

or, equivalently,

$$
\left(h\left(w\left(t_{1}\right)\right)-h\left(w\left(t_{2}\right)\right)\right)\left(h\left(w\left(t_{1}\right)\right)+h\left(w\left(t_{2}\right)\right)-2\right) \geqslant 0 .
$$

Since $w\left(t_{1}\right)<w\left(t_{2}\right)$ and $h$ is strictly decreasing, the required conclusion follows.

Proof of Corollary 3.8 Suppose for contradiction that $s_{0} \in\left(s_{1}, s_{2}\right)$ is a strict local minimum of $w$. Then $w^{\prime}\left(s_{0}\right)=0$. Let $\tilde{s} \in\left(s_{0}, s_{2}\right)$ be such that

$$
w(\tilde{s})=\max \left\{w(s): s \in\left[s_{0}, s_{2}\right]\right\} .
$$

Then there exist $t_{1}, t_{2}$ with $s_{0} \leqslant t_{1}<t_{2} \leqslant \tilde{s}$ such that $w^{\prime}\left(t_{1}\right)=w^{\prime}\left(t_{2}\right)=0$ and $0 \not \equiv w^{\prime} \geqslant 0$ on $\left(t_{1}, t_{2}\right)$. Now Theorem 3.7 yields $h\left(w\left(t_{1}\right)\right)+h\left(w\left(t_{2}\right)\right) \geqslant 2$, which contradicts the fact that $h\left(w\left(t_{1}\right)\right)<1$ and $h\left(w\left(t_{2}\right)\right)<1$. Hence no strict local minima of $w$ in $\left(s_{1}, s_{2}\right)$ exist.

Proof of Theorem 3.9 Since $\vartheta$ is continuous, $2 \pi$-periodic, and has zero mean, $\mathcal{R}(\vartheta)$ is a compact interval which contains 0 . We shall prove that $\pi / 2 \notin \mathcal{R}(\vartheta)$ and $-\pi / 2 \notin \mathcal{R}(\vartheta)$.

Suppose that $\pi / 2 \in \mathcal{R}(\vartheta)$. Then there exist $t_{1}, t_{2}$ with $t_{1}<t_{2}$ such that $\vartheta\left(t_{1}\right)=0, \vartheta\left(t_{2}\right)=\pi / 2$, and $0<\vartheta<\pi / 2$ on $\left(t_{1}, t_{2}\right)$. It follows from (4.4) using (4.3) that

$$
t \mapsto \frac{h^{2}(w(t))}{2}-h(w(t)) \cos \vartheta(t) \quad \text { is decreasing on }\left(t_{1}, t_{2}\right),
$$

so that

$$
\frac{h^{2}\left(w\left(t_{1}\right)\right)}{2}-h\left(w\left(t_{1}\right)\right) \geqslant \frac{h^{2}\left(w\left(t_{2}\right)\right)}{2}>0 .
$$

But this contradicts the assumption that $h(w(t)) \leqslant 2$ for all $t \in \mathbb{R}$. Hence $\pi / 2 \notin \mathcal{R}(\vartheta)$. 
Suppose now that $-\pi / 2 \in \mathcal{R}(\vartheta)$. Then there exist $t_{1}, t_{2}$ with $t_{1}<t_{2}$ such that $\vartheta\left(t_{1}\right)=-\pi / 2$, $\vartheta\left(t_{2}\right)=0$, and $-\pi / 2<\vartheta<0$ on $\left(t_{1}, t_{2}\right)$. It follows from (4.4) using (4.3) that

$$
t \mapsto \frac{h^{2}(w(t))}{2}-h(w(t)) \cos \vartheta(t) \quad \text { is increasing on }\left(t_{1}, t_{2}\right),
$$

so that

$$
0<\frac{h^{2}\left(w\left(t_{1}\right)\right)}{2} \leqslant \frac{h^{2}\left(w\left(t_{2}\right)\right)}{2}-h\left(w\left(t_{2}\right)\right),
$$

which again contradicts the assumption that $h(w(t)) \leqslant 2$ for all $t \in \mathbb{R}$. Hence $-\pi / 2 \notin \mathcal{R}(\vartheta)$. This completes the proof of Theorem 3.9

The proof of the following lemma is elementary, and is therefore omitted.

LEMmA 4.1 Let $j:(-\infty, 0) \rightarrow \mathbb{R}$ be a function of class $C^{1}$ with $j(x) \rightarrow \infty$ as $x \nearrow 0$, and for which there exists $\delta<0$ such that $j$ is convex on $[\delta, 0)$. Then for every $\alpha \in(-\infty, \delta]$ there exists $\beta \in[\delta, 0)$ such that

$$
j(x)+j^{\prime}(x)(y-x) \leqslant j(y) \quad \text { for all } x \in[\beta, 0) \text { and } y \in[\alpha, 0) .
$$

Proof of Theorem 3.3. Let $\delta$ be as in the statement of the theorem. Applying Lemma 4.1 with $j:=-\log h$ and $\alpha:=\min \mathcal{R}(w)$ yields $\beta \in[\delta, 0)$ such that, for all $t$ with $w(t) \in[\beta, 0)$ and for all $s \in \mathbb{R} \backslash \mathcal{N}$,

$$
\log h(w(t))+\left(h^{\prime}(w(t)) / h(w(t))\right)(w(s)-w(t))-\log h(w(s)) \geqslant 0 .
$$

Let $J \subset \mathbb{R} \backslash \mathcal{N}$ be a non-trivial interval such that $w(t) \in[\beta, 0)$ for all $t \in J$. It follows from (4.1) and 4.11) that, for all $t \in J$,

$$
\vartheta^{\prime}(t)-\frac{h^{\prime}(w(t))}{h(w(t))}\left(\frac{\cos \vartheta(t)}{h(w(t))}-1\right) \geqslant 0 .
$$

Note also that, for all $t \in J$,

$$
h^{\prime}(w(t)) \leqslant 0 .
$$

The burden of the proof of Theorem 3.3 is carried by the following lemma.

LEMMA 4.2 Let $w$ be a solution of 2.5 which satisfies 2.7 and for which $\mathcal{N} \neq \emptyset$.

If $d \in \mathbb{R} \backslash \mathcal{N}$ and $b \in \mathcal{N}$ are such that $(d, b) \subset \mathbb{R} \backslash \mathcal{N}$ and (4.12), (4.13) hold on $[d, b)$, then $\cos \vartheta>0$ on $[d, b)$.

Similarly, if $a \in \mathcal{N}$ and $c \in \mathbb{R} \backslash \mathcal{N}$ are such that $(a, c) \subset \mathbb{R} \backslash \mathcal{N}$ and 4.12, 4.13) hold on $(a, c]$, then $\cos \vartheta>0$ on $(a, c]$.

Assuming for the moment that Lemma 4.2 is true, we show how the proof of Theorem 3.3 follows.

Suppose that we are in the situation (i). Then (4.12) and 4.13 hold on $(a, b)$. Let $t_{0} \in(a, b)$ be such that

$$
w\left(t_{0}\right)=\min \{w(t): t \in[a, b]\} .
$$

Then (4.12) and 4.13 hold on each of the intervals $\left(a, t_{0}\right]$ and $\left[t_{0}, b\right)$. The first part of Lemma 4.2 applied with $d:=t_{0}$ yields $\cos \vartheta>0$ on $\left[t_{0}, b\right)$, while the second part of that lemma applied with $c:=t_{0}$ yields $\cos \vartheta>0$ on $\left(a, t_{0}\right]$. The conclusion follows. 
In the situation (ii), the conclusion is immediate from the first part of Lemma 4.2, and in the situation (iii) it follows from the second part of that lemma.

This completes the proof of Theorem 3.3 , provided that Lemma 4.2 is true.

Lemma 4.2 also leads to a quick proof of Theorem 3.2 .

Proof of Theorem 3.2 Since $h$ satisfies 3.1 , it follows that 4.12 and 4.13 hold on $\mathbb{R} \backslash \mathcal{N}$. If $\mathcal{N}=\emptyset$, then the required result follows easily (see [8, the sentence following (4.28)]). If $\mathcal{N} \neq \emptyset$, then it is immediate from Lemma 4.2 that $\cos \vartheta>0$ on $\mathbb{R} \backslash \mathcal{N}$ and therefore, by (2.10b), $w$ satisfies (3.3) as required.

It now remains to prove Lemma 4.2 .

Proof of Lemma 4.2 We prove only the first part of the lemma, since the second part follows by entirely similar arguments. The proof ultimately rests on an application of the following classical theorem in the global differential geometry of plane curves (see e.g. Amann [1, Theorem 24.15, p. 340] and the references therein) to a suitably devised Jordan curve.

THEOREM 4.3 Let $\sigma:[a, b] \rightarrow \mathbb{C}$ be a parametrization of a Jordan curve, where $\sigma$ is a function of class $C^{1}$ with $\sigma^{\prime}(a)=\sigma^{\prime}(b)$ and $\left|\sigma^{\prime}\right|>0$ on $[a, b]$. Let $\phi:[a, b] \rightarrow \mathbb{R}$ be a continuous function such that

$$
\sigma^{\prime}(t)=\left|\sigma^{\prime}(t)\right| \exp \{i \phi(t)\} \quad \text { for all } t \in[a, b] .
$$

Then $\phi(b)-\phi(a)$ equals either $2 \pi$ or $-2 \pi$.

We also use the following lemmas [8, Proof of Lemma 4.16 and Proof of Lemma 4.17].

LEMma 4.4 Let $[d, b) \subset \mathbb{R} \backslash \mathcal{N}$ and suppose that 4.12) and 4.13 hold on $[d, b)$. Let $e \in[d, b)$. If $\ell \in \mathbb{Z}$ is such that $\vartheta(e) \geqslant \ell \pi+\pi / 2$, then

$$
\vartheta(t)>\ell \pi+\pi / 2 \text { for all } t \in(e, b) .
$$

LEMma 4.5 Let $[d, b) \subset \mathbb{R} \backslash \mathcal{N}$ and suppose that (4.12) and (4.13) hold on $[d, b)$. Let $f \in[d, b)$ be such that $\cos \vartheta(f) \leqslant 0$. If $m \in \mathbb{Z}$ is such that

$$
2 m \pi+\pi / 2 \leqslant \vartheta(f) \leqslant 2 m \pi+3 \pi / 2,
$$

then there exists $g \in[f, b)$ such that $\vartheta$ is strictly increasing on $[f, g]$ and

$$
\vartheta(g)=2 m \pi+3 \pi / 2 \text {. }
$$

Let

$$
\widetilde{\mathcal{S}}:=\{(t+\mathcal{C} w(t), w(t)): t \in \mathbb{R}\},
$$

which by assumption is a non-self-intersecting curve. (The curve $\widetilde{\mathcal{S}}$ is obtained from the curve $\mathcal{S}$ given by 2.6 by reflection with respect to a vertical line.) Observe that, for almost all $t, \vartheta(t)$ is the angle between the tangent to $\widetilde{\mathcal{S}}$ at $(t+\mathcal{C} w(t), w(t))$ and the horizontal.

To prove Lemma 4.2 , we argue by contradiction and assume that there exists $f \in[d, b)$ with $\cos \vartheta(f) \leqslant 0$, which means

$$
\vartheta(f) \in[2 m \pi+\pi / 2,2 m \pi+3 \pi / 2] \quad \text { for some } m \in \mathbb{Z} .
$$


Since $w(d)=\beta$ and $w(t) \geqslant \beta$ for all $t \in[d, b)$, it follows that $w^{\prime}(d) \geqslant 0$, so that

$$
\vartheta(d) \in[2 k \pi,(2 k+1) \pi] \quad \text { for some } k \in \mathbb{Z} .
$$

Lemma 4.4 shows that $\vartheta(f)>2 k \pi-\pi / 2$, and so it follows from 4.15 that necessarily

$$
m \geqslant k
$$

Let $g \in(f, b)$ be given by Lemma 4.5 , so that

$$
\vartheta(g)=2 m \pi+3 \pi / 2 .
$$

Let $E<0$ be such that

$$
E>\max \{w(t): t \in[d, g]\},
$$

and let $p \in(g, b)$ be such that

$$
w(p)=E \quad \text { and } \quad w(t)<E \quad \text { for all } t \in(g, p) .
$$

It follows that $w^{\prime}(p) \geqslant 0$, and hence there exists $n \in \mathbb{Z}$ such that

$$
\vartheta(p) \in[2 n \pi,(2 n+1) \pi]
$$

It follows from Lemma 4.4 that

$$
n \geqslant m+1 \text {. }
$$

Let $\gamma: \mathbb{R} \rightarrow \mathbb{C}$ be given by

$$
\gamma(t):=t+\mathcal{C} w(t)+i w(t) \quad \text { for all } t \in \mathbb{R},
$$

and let $\tilde{\gamma}:[d, p] \rightarrow \mathbb{C}$ be the restriction of $\gamma$ to $[d, p]$. It is obvious that, for some $q_{1}, q_{2} \in \mathbb{R}$ with $q_{1}<d, p<q_{2}$, one can construct a function $\hat{\gamma}:\left[q_{1}, q_{2}\right] \rightarrow \mathbb{C}$, where

$$
\hat{\gamma}(q):=u(q)+i v(q) \quad \text { for all } q \in\left[q_{1}, q_{2}\right],
$$

such that $\hat{\gamma}$ is an extension of $\tilde{\gamma}$, and it has the following additional properties:

$$
\begin{aligned}
& u, v:\left[q_{1}, q_{2}\right] \rightarrow \mathbb{R} \quad \text { are of class } C^{1}, \\
& u^{\prime}(q)^{2}+v^{\prime}(q)^{2}>0 \quad \text { for all } q \in\left[q_{1}, q_{2}\right], \\
& v^{\prime} \geqslant 0 \quad \text { on }\left[q_{1}, d\right] \cup\left[p, q_{2}\right], \\
& v^{\prime}\left(q_{1}\right)=v^{\prime}\left(q_{2}\right)=1, \\
& u^{\prime}\left(q_{1}\right)=u^{\prime}\left(q_{2}\right)=0 .
\end{aligned}
$$

Let $F$ be such that

$$
F<\min \left\{u(q): q \in\left[q_{1}, q_{2}\right]\right\} .
$$

Let $\mathcal{A}_{1}$ be the semicircle having as diameter the segment joining the points $\left(F, v\left(q_{1}\right)\right)$ and $\left(u\left(q_{1}\right), v\left(q_{1}\right)\right)$, and situated below this segment. Let $\mathcal{A}_{2}$ be the semicircle having as diameter the segment joining the points $\left(u\left(q_{2}\right), v\left(q_{2}\right)\right)$ and $\left(F, v\left(q_{2}\right)\right)$, and situated above this segment. 
Let $r_{1}, r_{2}$ with $r_{1}<q_{1}, r_{2}>q_{2}$ and consider a $C^{1}$ function $\gamma_{*}:\left[r_{1}, r_{2}\right] \rightarrow \mathbb{C}$ which is an extension of $\hat{\gamma}$ such that

$$
\begin{aligned}
& \gamma_{*}\left(r_{1}\right)=F+i v\left(q_{1}\right), \quad \gamma_{*}\left(r_{2}\right)=F+i v\left(q_{2}\right), \\
& \gamma_{*}^{\prime}\left(r_{1}\right)=\gamma_{*}^{\prime}\left(r_{2}\right)=-i, \quad\left|\gamma_{*}^{\prime}(r)\right|>0 \text { for all } r \in\left[r_{1}, r_{2}\right], \\
& \left.\gamma_{*}\right|_{\left[r_{1}, q_{1}\right]} \text { is an injective parametrization of } \mathcal{A}_{1}, \\
& \left.\gamma_{*}\right|_{\left[q_{2}, r_{2}\right]} \text { is an injective parametrization of } \mathcal{A}_{2} .
\end{aligned}
$$

Let $\tilde{r}_{2}:=r_{2}$ and $\tilde{r}_{1}$ with $\tilde{r}_{1}<r_{1}$ be such that $\gamma_{*}$ has an extension to $\left[\tilde{r}_{1}, \tilde{r}_{2}\right]$ as a $C^{1}$ function such that

$$
\begin{aligned}
\gamma_{*}\left(\tilde{r}_{1}\right) & =F+i v\left(q_{2}\right), \\
\gamma_{*}^{\prime}(r) & =-i \quad \text { for all } r \in\left[\tilde{r}_{1}, r_{1}\right] .
\end{aligned}
$$

It is very easy to prove that $\gamma_{*}:\left[\tilde{r}_{1}, \tilde{r}_{2}\right] \rightarrow \mathbb{C}$ constructed above provides a parametrization of a Jordan curve with a continuously varying tangent. Let us write

$$
\gamma_{*}^{\prime}(r)=\left|\gamma_{*}^{\prime}(r)\right| \exp \left\{i \vartheta_{*}(r)\right\} \quad \text { for all } r \in\left[\tilde{r}_{1}, \tilde{r}_{2}\right],
$$

where $\vartheta_{*}:\left[\tilde{r}_{1}, \tilde{r}_{2}\right] \rightarrow \mathbb{R}$ is a continuous function which extends $\vartheta:[d, g] \rightarrow \mathbb{R}$. It follows from (4.16, , 4.18) and 4.20) that

$$
\vartheta_{*}\left(q_{1}\right)=2 k \pi+\pi / 2, \quad \vartheta_{*}\left(q_{2}\right)=2 n \pi+\pi / 2 .
$$

Using (4.21) and 4.22) we deduce that

$$
\vartheta_{*}\left(\tilde{r}_{1}\right)=2 k \pi-\pi / 2, \quad \vartheta_{*}\left(\tilde{r}_{2}\right)=2 n \pi+3 \pi / 2 .
$$

Therefore

$$
\vartheta_{*}\left(\tilde{r}_{2}\right)-\vartheta_{*}\left(\tilde{r}_{1}\right)=2(n-k) \pi+2 \pi,
$$

where, by (4.17) and (4.19),

$$
n-k \geqslant 1 \text {. }
$$

But the validity of (4.23) with (4.24) is in contradiction to Theorem 4.3 . This completes the proof of Lemma 4.2 .

\section{Acknowledgements}

I am extremely grateful to Professor J. F. Toland for many stimulating discussions on the topics of this work. Some of the results reported here were obtained during my $\mathrm{PhD}$ studies, when I was the recipient of a University of Bath Studentship and an ORS Award. Further results were obtained while I was visiting the Mittag-Leffler Institute in Stockholm, whose hospitality and support are gratefully acknowledged. 


\section{REFERENCES}

1. Amann, H. Ordinary Differential Equations. de Gruyter, Berlin (1990). Zbl 0708.34002 MR 1071170

2. Amick, C. J., Fraenkel, L. E., \& Toland, J. F. On the Stokes conjecture for the wave of extreme form. Acta Math. 148 (1982), 193-214. Zbl 0495.76021 MR 0666110

3. Duren, P. L. Theory of $H^{p}$ Spaces. Academic Press, New York (1970) and Dover, Mineola (2000). Zbl 0215.20203 MR 0268655

4. Koosis, P. Introduction to $H_{p}$ Spaces. 2nd ed., Cambridge Univ. Press, Cambridge (1998). Zbl 1024.30001 MR 1669574

5. Pichler-Tennenberg, A. K. On an equation related to Stokes waves. PhD Thesis, University of Bath, 2003, http://www.maths.bath.ac.uk/ jft/Papers/alex.pdf.

6. Plotnikov, P. I. A proof of the Stokes conjecture in the theory of surface waves. Dinamika Splosh. Sredy 57 (1982), 41-76 (in Russian); English transl.: Stud. Appl. Math. 3 (2002), 217-244. Zbl 0522.76017 MR 1883094

7. Plotnikov, P. I., \& Toland, J. F. Convexity of Stokes waves of extreme form. Arch. Ration. Mech. Anal. 171 (2004), 349-416. Zbl 1064.76017 MR 2038344

8. Shargorodsky, E., \& Toland, J. F. Bernoulli free-boundary problems. Mem. Amer. Math. Soc. (2007), to appear; http://www.maths.bath.ac.uk/ jft/Papers/memoir.pdf.

9. Spielvogel, E. R. A variational principle for waves of infinite depth. Arch. Ration. Mech. Anal. 39 (1970), 189-205. Zbl 0229.76012 MR 0268742

10. Toland, J. F. Stokes waves. Topol. Methods Nonlinear Anal. 7 (1996), 1-48 \& 8 (1997), 413-414. Zbl 0897.35067 MR 1422004 1483638

11. Toland, J. F. On a pseudo-differential equation for Stokes waves. Arch. Ration. Mech. Anal. 162 (2002), 179-189. Zbl 1028.35126 MR 1897380

12. Varvaruca, E. Singularities of Bernoulli free boundaries, Comm. Partial Differential Equations 31 (2006), 1451-1477. Zbl 1111.35137| MR 2273961 